\title{
Activation tagging in Salvia miltiorrhiza can cause increased leaf size and accumulation of tanshinone I and IIA in its roots
}

\author{
Hsin-Shueh Ho ${ }^{1}$, Rishi Kishore Vishwakarma ${ }^{1}$, Emily Chin-Fun Chen ${ }^{1}$ and Hsin-Sheng Tsay ${ }^{1,2^{*}}$
}

\begin{abstract}
Background: Salvia miltiorrhiza Bunge (Danshen), an important herb in traditional Chinese medicine, is commonly used for treatment of cardiovascular diseases. One of the major bioactive constituents of Danshen, diterpenoid tanshinone, has been proved with pharmacological properties and have the potential to be a new drug candidate against various diseases. In our previous study, we have established an activation tagging mutagenesis (ATM) population of callus lines of S. miltiorrhiza Bunge by Agrobacterium- mediated transformation.

Results: In the present study, we have identified ATM transgenic Salvia plant (SH41) with different leaf morphology and more tanshinones in its roots. The transgenic background of SH41 was identified by PCR (using hptll primers) and Southern blots. PCR analysis showed a single band of hptll gene and Southern blot analysis showed single insertion in SH41. External appearance of ATM transgenic SH41 was observed with broader leaves comparing to non-transformed plants. More healthy trichomes as well as bigger and wobbly guard cells and stomata were observed in SH41 by scanning electron microscopy (SEM). Quantitative analysis of active compounds in SH41 roots revealed a significant increase in tanshinone I (3.7 fold) and tanshinone IIA ( 2 fold) contents as compared to the wild plant.
\end{abstract}

Conclusions: We have generated an activation tagged transgenic Salvia plant (SH41) with different leaf morphology and high diterpenes content in its roots. The increased amount of tanshinones in SH41 will definitely offer a route for maximizing the benefits of this plant in traditional Chinese herbal medicines. The present report may also facilitate the application of ATM for genetic manipulation of other medicinal crops and subsequent improved metabolite contents.

Keywords: Activation-tagging; HPLC; Tanshinones; Salvia miltiorrhiza; Scanning electron microscopy

\section{Background}

Salvia miltiorrhiza Bunge (Danshen) is a recognized traditional Chinese medicine and is broadly used for the treatment of various cardiovascular and cerebrovascular diseases. This wide range of medicinal uses is mainly due to its property of stimulating blood circulation and removing blood stasis (Zhou et al. 2012). Danshen contained water soluble phenolics including danshensu, protocatechuic aldehyde, protocatechuic acid, caffeic acid, rosmarinic acid and salvianolic acid A \& B as well as lipid soluble diterpene quinones: cryptotranshinone, transhinone I and transhinone IIA (Liu et al. 2007). Phenolics and quinones have alike

\footnotetext{
* Correspondence: hstsay@cyut.edu.tw

'Department of Applied Chemistry, Chaoyang University of Technology, 168, Jifong E Road, Taichung, Wufong 41349, Taiwan ${ }^{2}$ Department of Agronomy, National Chung Hsing University, Taichung, Taiwan
}

individual pharmacological properties such as antioxidant, anti-apoptosis and vasodilation (Zhou et al. 2005; Lam et al. 2007; Lam et al. 2008a; Wang et al. 2011a).

Many experimental and clinical investigations have reported that tanshinones and their derivatives can prevent or slow the development of a various diseases including hypoxia/reoxygenation injury, cardiovascular diseases, cancer, neonatal hypoxic ischemic encephalopathy, hepatic fibrosis as well as neuro degenerative diseases (Wu et al. 1993; Yagi et al. 1994; Yoon et al. 1999; Takahashi et al. 2002; Han et al. 2008; Shu et al. 2010).

Due to its extensive medicinal values, S. miltiorrhiza has been studied by various research groups over the world, especially in biotechnological and in vitro propagation field. Through several strategies like genetic manipulation of biosynthetic pathway via genetic transformation (Chen et al. 1997; Lee et al. 2008; Kai et al. 2011), hairy 
root cultures (Hu et al. 1993), use of plant growth regulators and elicitors (Wu et al. 2003; Zhang et al., 2004; Ge and Wu 2005a, 2005b; Gupta et al. 2011), success has been reported in enhancing the production of secondary metabolites in S. miltiorrhiza.

Although, several reports represented the micropropagation and genetic transformation methods for S. miltiorrhiza by other means (Zhang et al. 1995; Chen et al. 1997; Zhang et al. 1997; Yan and Wang 2007; Lee et al. 2008), but there is a limited report available on activation tagging mutagenesis. Therefore, the overall objective of the current research was to develop and investigate the effect of ATM on morphological as well as histological changes and tanshinones content in transgenic Salvia plant.

\section{Methods}

Plant material

S. miltiorrhiza used for this study was collected from Zhengzhou City, Henan Province Jiyuan County mountains, China. Activation tagged mutagenic (ATM) Salvia plants are maintained at greenhouse in Chaoyang University of Technology.

\section{Gene constructs and bacterial strain}

A. tumefaciens strain EHA105 harboring a binary vector pTAG8 (Hsing et al. 2007; Chen et al. 2009; Tsay et al. 2012) was used for the genetic transformation of S. miltiorrhiza. The T-DNA region of the binary vector contained a selectable marker coding for the hygromycin phosphotransferase (hptII) gene under control of CaMV $35 \mathrm{~S}$ promoter (provided by Dr. Su-May Yu, Academia Sinica, Taipei, Taiwan).

\section{Genetic transformation and confirmation of ATM insertion in Salvia plant}

Salvia transgenic raised by Lee et al. (2008) was used for this study. ATM transgenic S. miltiorrhiza plant (SH41) with an altered morphological appearance was investigated. The transformation was confirmed by PCR detection of hygromycin phsphotransferase gene (hptII) gene using genomic DNA and gene specific primers $h p t \mathrm{II} F$ 5' -GTCGTGGCGATCCTGCAAGC-3' and hptII R 5' -CC TGCGGGTAAATAGCTGCGC-3'. Total genomic DNA was isolated from young leaves of SH41 and control plants using ZR plant/seed DNA MiniPrep kit (Zymo Research). The PCR reaction contained $50 \mathrm{ng}$ genomic DNA, $0.2 \mathrm{mM}$ dNTPs mix, $500 \mathrm{nM}$ each forward and reverse primers, and $1 \mathrm{U}$ of Taq DNA polymerase. The PCR was carried out in a thermal cycler (BIO-RAD, USA) under the following conditions: 1 cycle of $94^{\circ} \mathrm{C}$ (5 min), 35 cycles of $94^{\circ} \mathrm{C}(30 \mathrm{~s})$, $55^{\circ} \mathrm{C}(30 \mathrm{~s})$ and $72^{\circ} \mathrm{C}(1 \mathrm{~min})$, and final extension at $72^{\circ} \mathrm{C}$ (7 min). The plasmid pTAG8 was used as a positive control. The amplification products were analyzed on $1 \%$ agarose gel by electrophoresis and visualized under UV light after ethidium bromide staining.

The insertion of the gene was further confirmed by Southern blot analysis. Genomic DNA of SH41 and control plants was isolated using the modified cetyl trimethyl ammonium bromide (CTAB) method (Doyle and Doyle 1987). Isolated genomic DNA (15 $\mu \mathrm{g})$ was digested overnight with restriction enzymes PstI, HindIII and EcoRI in appropriate buffer at $37^{\circ} \mathrm{C}$. The digested DNA was electrophoresed on $0.8 \%$ agarose gel in $1 \mathrm{X}$ Tris-acetate/EDTA buffer. The gel was then treated with dupurination, denaturation and neutralization buffers and DNA was blotted onto a positively charged nylon membrane (Bio-Rad) as described by Sambrook et al. (1989). A PCR-amplified hptII gene fragment was labelled with digoxigenin-9-dUTP using Dig High Prime Labeling and Detection Starter Kit I (Roche, USA) and used as a probe. After prehybridization for $2 \mathrm{~h}$ at $52^{\circ} \mathrm{C}$, labeled probe was added and kept for hybridization at the same temperature for $16 \mathrm{~h}$ in hybridization chamber. The membrane was subjected to a series of stringency washes (high stringency at $56^{\circ} \mathrm{C}$ ) to eliminate nonspecific probe binding. The signals were immuno-detected with antidigoxigenin-AP and visualized with the colorimetric substrates BCIP/NBT according to manufacturer's instruction.

\section{Scanning electron microscopy (SEM) analysis}

In order to investigate the differences observed in the morphology of the wild type (WT) and transgenic (SH41) Salvia plants, scanning electron microscopy (SEM) was performed. Saliva leaf and root samples were cut into an appropriate size, adhered to the metal base, frozen in liquid nitrogen and scanned in low-vacuum SEM (JEOL JSM-633OF) with chamber pressure of $30 \mathrm{~Pa}$ and an accelerated voltage $15 \mathrm{kV}$.

\section{Quantitative (HPLC) analysis}

High performance liquid chromatography (HPLC) was performed to detect the water soluble metabolites danshensu, protocatechuic acid, protocatechuic aldehyde, caffeic acid, rosmarinic acid, salvianolic acid A and salvinic acid B, as well as lipid soluble tanshinone I, tanshinone $\Pi \mathrm{A}$ and cryptotanshinone in roots. All the standards were obtained from Sigma and Formosa Kingstone Bioproducts International Corp.

First, extraction was optimized using different solvent system with commercially available Salvia plant roots (CK; purchased from Joint Pharmacy, Taichung City, Taiwan). For further analysis, $50 \mathrm{mg}$ dried roots of 4 months old transgenic (SH41) and wild type Salvia plants was finely ground by mortar pestle using liquid nitrogen and extracted with $10 \mathrm{ml}$ of $60 \%$ ethanol containing $0.05 \%$ formic acid by sonication for $10 \mathrm{~min}$ along with and commercially available (CK) Salvia root 
powder. The extracts were filtered through filter paper and vacuum dried. Further, the dried samples were dissolved in methanol/water (1:1), filtered through $0.22 \mu \mathrm{m}$ membrane and subjected to HPLC analysis. The HPLC system consisted of a pump (Hitachi L-2130), automatic injector (Hitachi L-2200) and Diode Array Detector (Hitachi L-2450). The column used was C18 (Waters; 5 um, $4.6 \times 250 \mathrm{~mm}$ ) and UV detection was carried out at $280 \mathrm{~nm}$. The mobile phase acetonitrile/water containing $0.05 \% \mathrm{H}_{3} \mathrm{PO}_{4}$ was pumped at a flow rate was $1.0 \mathrm{~mL} / \mathrm{min}$ and programmed as follows: $10 \%$ acetonitrile for $0 \mathrm{~min}$; $40 \%$ acetonitrile for $15 \mathrm{~min}$; $60 \%$ acetonitrile for $15 \mathrm{~min}$ and $75 \%$ acetonitrile for $30 \mathrm{~min}$. Dilutions (1.512, 3.125, $6.25,12.5,25,50,100 \mathrm{mg} / \mathrm{mL}$ ) of standard compounds were prepared and $10 \mu \mathrm{L}$ aliquots were subjected to HPLC. This was repeated thrice and calibration plots were constructed from the peak areas of standard compounds.

\section{Statistical analysis}

Each result shown in tables was the mean of at least three replicated experiments and the standard deviation $( \pm \mathrm{SD})$ value was calculated.

\section{Results}

Morphological investigation of activation tagged Salvia plant As shown in Figure 1, we can clearly see the morphological difference between SH41 and wild type plant.
SH41 plant exhibited larger sized leaves as well as thick and smaller petiole than wild type (Figure 1).

The microscopic (SEM) analysis showed that activation tag mutagenesis affected the morphological development of leaf tissues during growth. Cross section of leaf blades showed thickening of the palisade and the spongy tissues were slightly larger in SH41 as compared to wild type (Figures 2A, 2B). No significant differences were observed in the morphology of roots (Figures $2 \mathrm{C}$, 2D). Adaxial and abaxial surface of SH41 leaf showed more trichomes than WT with bigger and wobbly guard cells and stomata (Figures 2E, 2F, 2G, 2H). Two types of hairy assemblies were found in the microscopic observation of leaves, one similar to flagpole-shaped head (Figures 3A, 3B) and another bamboo shoot shaped (Figures $3 \mathrm{C}, 3 \mathrm{D}$ ), the latter is mostly deposited in the blade surface in the $\mathrm{SH} 41$.

\section{PCR identification and DNA blot analysis of ATM transgenic Salvia}

PCR analysis with hptII gene specific primers was carried out to confirm the presence of the activation tagging T-DNA insertion in the genome of the putative transformant. The 650 bp expected hptII fragment was found in the positive control (pTAG8 plasmid) as well as in transformed Salvia SH41. No amplification was observed in wild type plant (control) (Figure 4A).
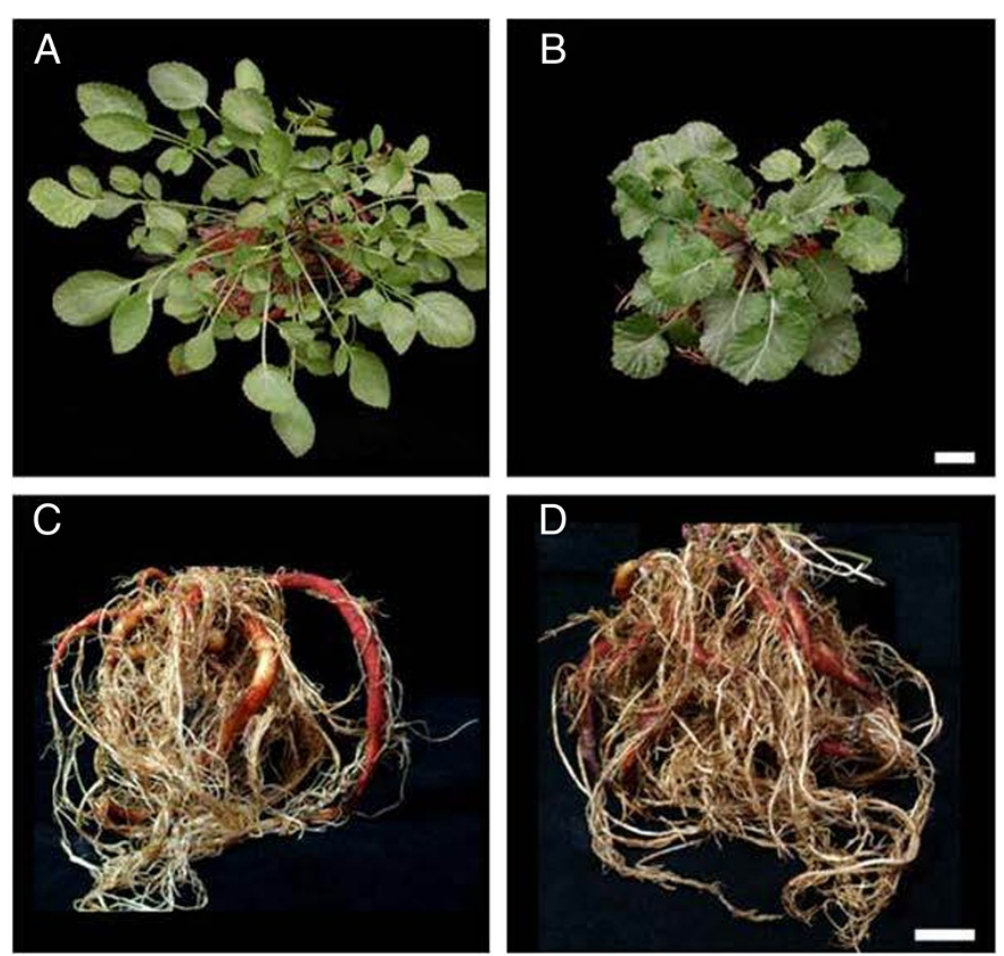

Figure 1 S. miltiorrhiza plant morphology. (A) Wild type (WT)- above ground. (B) SH41- above ground. (C) WT- roots. (D) SH41- roots. Scale $\mathrm{bar}=1 \mathrm{~cm}$. 


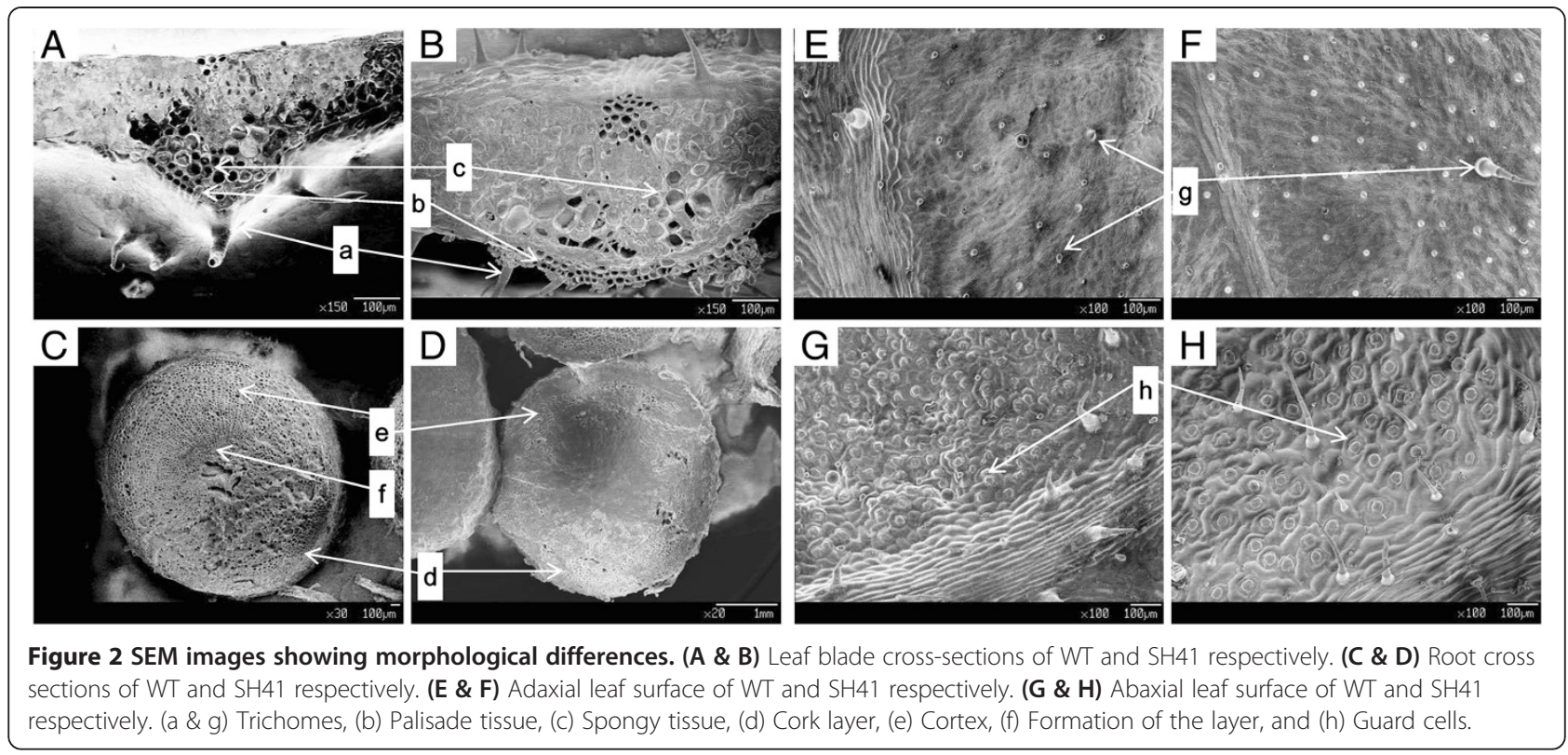

Southern hybridization analysis was performed in order to estimate the number of inserted loci. Figure $4 \mathrm{~B}$ showed the Southern hybridization pattern obtained with hptII probe. The result showed single insertion in SH41 and no band was observed in the non-transformed control plant (Figure 4B).

\section{Compound extraction and HPLC analysis}

For optimal extraction of active constituents from the Salvia plant, different solvent system was tested. The addition of formic acid improved the extraction efficiency of salvianolic acid B and Danshensu. As the result, $60 \%$ ethanol with $0.05 \%$ formic acid was the best solvent system (Table 1). The tanshinone I and tanshinone IIA detected in SH41 was 3.7 and 2 fold higher than wild type plant, respectively. These compounds were also greater than CK ( 2 fold and 3 fold higher, respectively). Salvianolic acid B was also found 1.4 and 2.4 fold higher in SH41 as compared to wild plant and CK, respectively. Other compounds like Danshensu, rosmarinic acid and

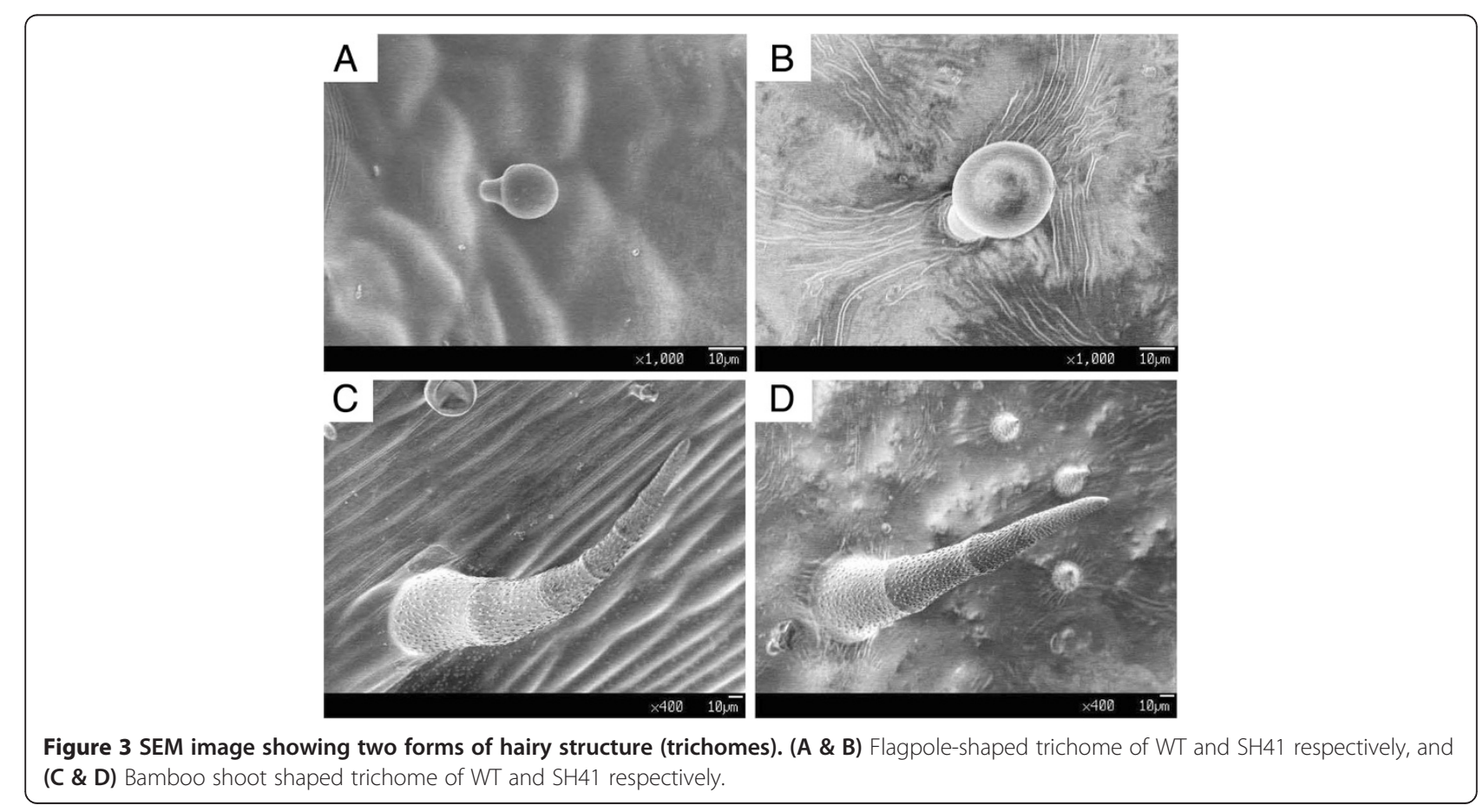




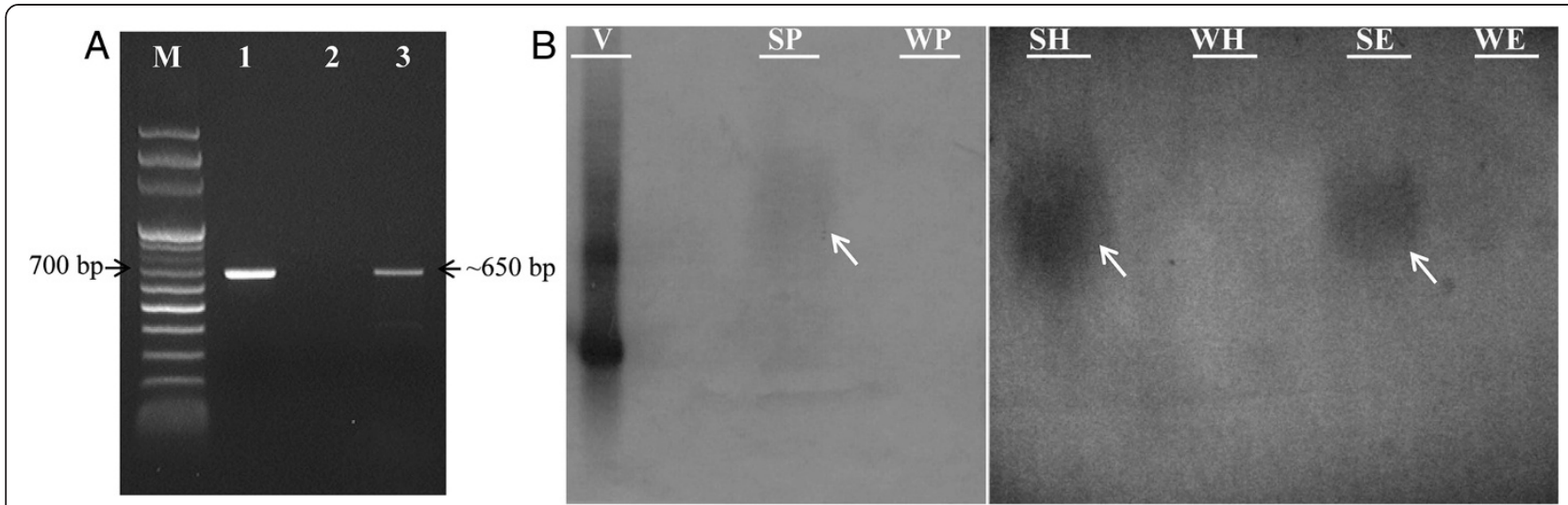

Figure 4 Molecular analysis of ATM transformed S. miltiorrhiza. (A) PCR analysis of transformerd Salvia plant. Genomic DNA was amplified using hptll gene specific primers. Approx. 650 bp amplification was observed in transformed plant. Lanes: (M) Molecular weight marker, (1) Positive control (pTAG8 plasmid), (2) Wild type Salvia; and (3) Active mutagenic Salvia plant (SH41). (B) Confirmation of activation-tagged S. miltiorrhiza Bunge by Southern blot analysis. Genomic DNA was extracted from leaves and hptll gene used as a probe. Hybridization was performed at 52 ${ }^{\circ} \mathrm{C}$. V- pTAG-8; SPSH41 and WP- WT digested by PSt I; SH- SH41 and WH-WT digested by Hind III; SE- SH41 and WE- WT digested by EcoR I. Arrows indicate hybridization signals.

salvianolic acid A were slightly higher in SH41, however, salvianolic acid A was less in SH41 as compared to CK. On the other hand, protocatechuic, protocatechuic aldehyde and caffeic acid were not detected in SH41 (Table 2).

\section{Discussion}

A new biotechnological method of mutagenesis, called activation tagging mutagenesis (ATM), has increased the understanding of development in various plants by intensifying the range of gene expressions and accumulation of secondary metabolites. This technique exploits the transferred DNA (T-DNA) from Agrobacterium tumefaciens to introduce a viral CaMV35S enhancer region randomly into the genome. The enhancer may dominantly "activate" and/or widen the pattern of expression of a gene that is near the enhancer of the introduced T-DNA (Hsing et al. 2007). It has been reported that the CaMV35S enhancer activate the expression of genes located upstream or downstream of T-DNA in Arabidopsis and rice (Ichikawa et al. 2003; Jeong et al. 2006). The insertion of an enhancer sequence in the vicinity of an endogenous gene can alter the transcriptional pattern of the gene, resulting in a mutant phenotype. Activation tagging has been undertaken extensively in a number of dicot and monocot plants (Michael and Anthony 2011).

Salvia miltiorrhiza (Danshen) is an important Chinese medicinal herb and diterpene tanshinones are its major active compounds. In previous studies, in vitro methods such as hairy root cultures (Hu et al. 1993; Ge and Wu 2005a; Yan et al. 2005), cell suspension cultures (Chen et al. 1997), addition of elicitors and optimization of media (Wu et al. 2003; Zhang et al. 2004; Ge and Wu 2005b; Gupta et al. 2011), have been reported. In our previous study, ATM transgenic callus lines of Salvia with greater tanshinones content were developed (Lee

Table 1 Different solvent extractions of Salvia miltiorrhiza root (commercially available plant)

\begin{tabular}{|c|c|c|c|c|c|c|}
\hline Compounds & $100 \% \mathrm{CH}$ & $70 \% \mathrm{CH}$ & $70 \% \mathrm{CH} / \mathrm{F}$ & $100 \% \mathrm{Et}$ & $60 \% \mathrm{Et}$ & $60 \% \mathrm{Et} / \mathrm{F}$ \\
\hline Danshensu & $0.63 \pm 0.06$ & $1.05 \pm 0.12$ & $1.22 \pm 0.02$ & $0.36 \pm 0.04$ & $0.98 \pm 0.04$ & $1.30 \pm 0.04$ \\
\hline Protocatechuic & $0.10 \pm 0.01$ & $0.13 \pm 0.01$ & $0.12 \pm 0.01$ & $0.09 \pm 0.03$ & $0.13 \pm 0.13$ & $0.14 \pm 0.14$ \\
\hline Protocatechuic aldehyde & $0.06 \pm 0.05$ & $0.06 \pm 0.01$ & $0.06 \pm 0.03$ & $0.06 \pm 0.02$ & $0.06 \pm 0.06$ & $0.06 \pm 0.06$ \\
\hline Caffeic acid & $0.06 \pm 0.05$ & $0.06 \pm 0.02$ & $0.06 \pm 0.03$ & $0.05 \pm 0.02$ & $0.06 \pm 0.06$ & $0.06 \pm 0.06$ \\
\hline Rosmarinic acid & $0.65 \pm 0.07$ & $1.08 \pm 0.08$ & $1.20 \pm 0.02$ & $0.36 \pm 0.03$ & $0.96 \pm 0.02$ & $1.21 \pm 0.04$ \\
\hline Salvianolic acid B & $3.35 \pm 0.09$ & $5.43 \pm 0.04$ & $5.97 \pm 0.08$ & $1.68 \pm 0.08$ & $4.87 \pm 0.07$ & $6.08 \pm 0.06$ \\
\hline Salvianolic acid A & $0.25 \pm 0.03$ & $0.36 \pm 0.04$ & $0.39 \pm 0.04$ & $0.16 \pm 0.01$ & $0.33 \pm 0.03$ & $0.41 \pm 0.02$ \\
\hline Cryptotanshinone & $0.03 \pm 0.01$ & $0.06 \pm 0.02$ & $0.07 \pm 0.02$ & $0.07 \pm 0.03$ & $0.06 \pm 0.01$ & $0.07 \pm 0.02$ \\
\hline Tanshinone I & $0.08 \pm 0.03$ & $0.10 \pm 0.04$ & $0.11 \pm 0.03$ & $0.18 \pm 0.04$ & $0.07 \pm 0.02$ & $0.14 \pm 0.05$ \\
\hline Tanshinone IIA & $0.04 \pm 0.01$ & $0.07 \pm 0.02$ & $0.08 \pm 0.02$ & $0.15 \pm 0.04$ & $0.07 \pm 0.01$ & $0.14 \pm 0.03$ \\
\hline
\end{tabular}

$\mathrm{CH}$, Methanol; $\mathrm{CH} / F$, Methanol with $0.05 \%$ formic acid; $E t$, Ethanol; $E t / F$, Ethanol with $0.05 \%$ formic acid. Data expressed as mean $\pm \mathrm{SD}$ for 3 repeats (mg/g). 
Table 2 HPLC-UV detection of Salvia roots extracted with $60 \%$ ethanol containing $0.05 \%$ formic acid for seven water-soluble and three fat-soluble ingredients, $(n=3)$ (mean \pm SD; $\mathbf{m g} / \mathrm{g}$ extract)

\begin{tabular}{lccc}
\hline Constituents & WTR & SHR & CK \\
\hline Danshensu & $0.19 \pm 0.07$ & $0.28 \pm 0.08$ & $1.30 \pm 0.17$ \\
Protocatechuic & ND & ND & $0.14 \pm 0.04$ \\
Protocatechuic aldehyde & $0.08 \pm 0.01$ & ND & $0.06 \pm 0.01$ \\
Caffeic acid & ND & ND & $0.06 \pm 0.04$ \\
Rosmarinic acid & $2.11 \pm 0.21$ & $2.87 \pm 0.74$ & $1.21 \pm 0.47$ \\
Salvianolic acid B & $10.44 \pm 0.75$ & $14.45 \pm 0.37$ & $6.08 \pm 0.39$ \\
Salvianolic acid A & $0.12 \pm 0.07$ & $0.16 \pm 0.09$ & $0.41 \pm 0.04$ \\
Cryptotanshinone & $0.59 \pm 0.13$ & $0.34 \pm 0.08$ & $0.07 \pm 0.05$ \\
Tanshinone I & $0.08 \pm 0.01$ & $0.30 \pm 0.05$ & $0.14 \pm 0.06$ \\
Tanshinone IIA & $0.20 \pm 0.09$ & $0.42 \pm 0.09$ & $0.14 \pm 0.04$ \\
\hline
\end{tabular}

WTR Wild type root, SHR SH41 root, CK Commercially available Salvia miltiorrhiza root, ND Non detectable.

et al. 2008). In the present study, one of the 4 months old ATM transgenic (SH41) with an altered phenotype was identified and subjected to quantitative analysis of medicinally important ingredients. Insertion of T-DNA was confirmed by PCR with hygromycin phosphotransferase (hptII ) gene specific primers and Southern blot analysis. PCR analysis showed amplification of hptII gene which confirmed the presence of an activation tagging T-DNA in SH41. DNA blot analysis showed single copy insertion of transgene in SH41. Single or low-copy integration of transgenes through Agrobacterium have been observed in several plant species (Find et al. 2005; Maghuly et al. 2006; Tsay et al. 2012). Microscopic (SEM) analysis showed adaxial and abaxial surface of transgenic Salvia leaves has more trichomes as well as bigger and wobbly guard cells and stomata. In contrast, wild type plant showed the less number of trichomes and smaller guard cells and stomata. Genetic modification in plants leads to change in morphology. Ectopic expression of gene in Arabidopsis showed different morphology and abnormal flowers containing wrinkled organs (Liu et al. 2008). Weigel et al. (2000) reported that the $35 \mathrm{~S}$ enhancer element might increase the expression of nearby genes without altering the original expression pattern. Since, incorporation of CaMV35S enhancers of pTAG8 is random in the genome, so changes in leaf morphology of SH41 is may be due to the activation of genes responsible for leaf development, which remains to be confirmed.

Based on previous studies, phenolic acids and diterpene contents vary with different solvent systems and extraction methods. In the present study, the combinations of methanol and ethanol with water were used to recover greater yield of both water as well as lipid soluble constituents of Salvia roots by sonication. It was observed that, $60 \%$ ethanol containing $0.05 \%$ formic acid was the best extraction system and the addition of formic acid increased the extraction efficiency (Table 1). Although, the effect of temperature on extraction were reported in other studies, such as highest content of salvianolic acid B $(48.37 \pm 1.21 \mathrm{mg} / \mathrm{g})$ was yielded in microwave assisted extraction with water (MAE-W) at $75^{\circ} \mathrm{C}$, or the highest contents of tanshinones including dihydrotanshinone, cryptotanshinone, tanshinone I, and tanshinone IIA were obtained in MAE-W at $100^{\circ} \mathrm{C}$ (Zhou et al. 2012), but we needed a method to extract salvianolic acid B and tanshinones simultaneously. Therefore, $60 \%$ ethanol containing $0.05 \%$ formic acid was the best choice for this study.

The active compound of S. miltiorrhiza, tanshinone, was proved with lots of biological activities. For instance, tanshinone I could enhance learning and memory (Kim et al. 2009), while tanshinone IIA has anti-oxidative (Wang et al. 2003; Yang et al. 2008), anti-inflammatory (Jang et al. 2006), anti-proliferative (Liu et al. 2006) and anti-tumor properties (Dong et al. 2007). Therefore, S. miltiorrhiza has been widely used to produce a number of traditional Chinese medicine preparations. However, because of low content, the supplement of tanshinone for the increasing needs of clinical applications has become a research attention. Therefore, development of transgenic Salvia with altered tanshinone content could overcome the shortage of traditional Chinese medicine preparations. Manipulating multiple genes at multiple control sites in desired metabolite production may cause metabolic flux alteration (Nims et al. 2006). The activation tagging has the ability to activate whole branches of biochemical pathways leading to the accumulation of several subclasses of natural product usually present in low concentration (Tani et al. 2004). In this study ATM transgenic of Salvia showed significant increase in diterpene tanshinone contents in roots as compared to wild plant. Tanshinone I, tanshinone IIA and salvianolic acid B were 3.7, 2 and 1.4 fold higher in SH41 as compared to wild plant. In other study it was reported that the over-expression of genes encoding 3-hydroxy-3methylglutaryl CoA reductase (SmHMGR), 1-deoxy-Dxylulose-5-phosphatesynthase $(S m D X S)$ and geranylgeranyl diphosphate synthase (SmGGPPS) in S. miltiorrhiza can significantly enhance the production of tanshinones in roots to levels higher than that of the control. S. miltiorrhiza plants over-expressing SmHMGR, SmGGPS and $S m D X S$ produced 2.1 to 5.7 fold higher tanshinones than control plants (Kai et al. 2011).

\section{Conclusions}

In conclusion, the current study provides good basement and helpful information for commercial large-scale production of tanshinones in the roots of Salvia by developing ATM transgenic lines with altered phenotype. Also, 
the extraction procedure and quantitative HPLC procedures were developed for determining liposoluble as well as water-soluble phenolic acid compounds.

\section{Abbreviations}

ATM: Activation tagging mutagenesis; HPLC: High performance liquid chromatography; hptll: Hygromycin phosphotransferase II gene; SEM: Scanning electron microscopy.

\section{Competing interests}

The authors declare that they have no competing interests.

\section{Authors' contributions}

Prof. HS Tsay designed experiment and reviewed the manuscript. Mr. HS Ho performed experiments. Dr. RK Vishwakarma prepared the manuscript. Prof. Emily CF Chen helped in analyzing data and proof reading of the manuscript. All authors read and approved the final manuscript.

\section{Acknowledgements}

The authors wish to thank Dr. Chiou-Ing Yuan (From Taiwan Agricultural Chemical and Toxic Substances Research Institute, Council of Agriculture) for providing SEM facility. This work was supported by National Science Council of Taiwan under the project grant NSC-97-2317-B-324-004.

\section{Received: 9 July 2013 Accepted: 5 September 2013}

Published: 24 September 2013

\section{References}

Chen H, Yuan JP, Chen F, Zhang YL, Song JY (1997) Tanshinone production in Titransformed Salvia miltiorrhiza cell suspension cultures. J Biotechnol 58:147-156

Chen ECF, Su YH, Kanagarajan S, Agrawal DC, Tsay HS (2009) Development of an activation tagging system for the basidiomycetous medicinal fungus Antrodia cinnamomea. Mycol Res 113:290-297

Dong XR, Dong JH, Peng G, Hou XH, Wu G (2007) Growth-inhibiting and apoptosis-inducing effects of Tanshinone IIA on human gastric carcinoma cells. J Huazhong Univ Sci Technol 27:706-709

Doyle JJ, Doyle $J$ (1987) A rapid DNA isolation procedure for small quantities of fresh leaf tissue. Phytochem Bull 19:11-15

Find JI, Charity JA, Grace LJ, Kritensen MMMH M, Krogstrup P, Walter C (2005) Stable genetic transformation of embryogenic cultures of Abies nordmanniana (Nordmann fir.) and regeneration of transgenic plants. In Vitro Cell Dev Biol Plant 41:725-730

Ge X, Wu J (2005a) Induction and potentiation of diterpenoid tanshinone accumulation in Salvia miltiorrhiza hairy roots by beta-aminobutyric acid. Appl Microbiol Biotechnol 68:183-188

Ge X, Wu J (2005b) Tanshinone production and isoprenoid pathways in Salvia miltiorrhiza hairy roots induced by $\mathrm{Ag}+$ and yeast elicitor. Plant Sci 168:487-491

Gupta SK, Liu RB, Liaw SY, Chan HS, Tsay HS (2011) Enhanced tanshinone production in hairy roots of 'Salvia miltiorrhiza Bunge' under the influence of plant growth regulators in liquid culture. Bot Stud 52:435-443

Han JY, Fan JY, Horie Y, Miura S, Cui DH, Ishii H, Hibi T, Tsuneki H, Kimura I (2008) Ameliorating effects of compounds derived from Salvia miltiorrhiza root extract on microcirculatory disturbance and target organ injury by ischemia and reperfusion. Pharmacol Ther 117:280-295

Hsing YI, Chen CG, Fan MJ, Lu PC, Chen KT, Lo SF, Sun PK, Ho SL, Lee KW, Wang YC, Huang WL, Ko SS, Chen S, Chen JL, Chung Cl, Lin YC, Hour AL, Wang YW, Chang YC, Tsai MW, Lin YS, Chen YC, Yen HM, Li CP, Wey CK, Tseng CS, Lai MH, Huang SC, Chen $\amalg$, Yu SM (2007) A rice gene activation/knockout mutant resource for high throughput functional genomics. Plant Mol Biol 63:351-364

Hu ZB, Alfermann AW (1993) Diterpenoid production in hairy roots cultures of Salvia miltiorrhiza. Phytochemistry 32:99-103

Ichikawa T, Nakazawa M, Kawashima M, Muto S, Gohda K, Suzuki K, Ishikawa A, Kobayashi H, Yoshizumi T, Tsumoto Y, Tsuhara Y, lizumi H, Goto Y, Matsui M (2003) Sequence database of 1172 T-DNA insertion sites in Arabidopsis activation-tagging lines that showed phenotypes in $\mathrm{T} 1$ generation. Plant J $36: 421-429$

Jang SI, Kim HJ, Kim YJ (2006) Tanshinone IIA inhibits LPS-induced NF-kB activation in RAW264.7cells: possible involvement of the NIK-IKK, ERK1/2, p38 and JNK pathways. Eur J Pharmacol 542:1-7
Jeong DH, An S, Park S, Kang HG, Park GG, Kim SR, Sim J, Kim YO, Kim MK, Kim SR, Kim J, Shin M, Jung M, An G (2006) Generation of a flanking sequencetag database for activation-tagging lines in japonica rice. Plant J 45:123-132 Kai G, Xu H, Zhou C, Liao P, Xiao J, Luo X, You L, Zhang L (2011) Metabolic engineering tanshinone biosynthetic pathway in Salvia miltiorrhiza hairy root cultures. Metab Eng 13:319-327

Kim DH, Kim S, Jeon SJ, Son KH, Lee S, Yoon BH, Cheong JH, Ko KH, Ryu JH (2009) Tanshinone I enhances learning and memory, and ameliorates memory impairment in mice via the extracellular signal-regulated kinase signalling pathway. Br J Pharmacol 158:1131-1142

Lam FF, Yeung JH, Chan KM, Or PM (2007) Relaxant effects of danshen aqueous extract and its constituent danshensu on rat coronary artery are mediated by inhibition of calcium channels. Vascul Pharmacol 46:271-277

Lam FF, Yeung JH, Chan KM, Or PM (2008a) Dihydrotanshinone, a lipophilic componentof Salvia miltiorrhiza (danshen), relaxes rat coronary artery by inhibition of calcium channels. J Ethnopharmacol 119:318-321

Lee CY, Agrawal DC, Wang CS, Yu SM, Chen JW, Tsay HS (2008) T-DNA activation tagging as a tool to isolate Salvia miltiorrhiza Bunge transgenic lines for higher yields of tanshinones. Planta Med 74:1-7

Liu JJ, Lin DJ, Liu PQ, Huang M, Li XD, Huang RW (2006) Induction of apoptosis and inhibition of cell adhesive and invasive effects by tanshinone IIA in acute promyelocytic leukemia cells in vitro. J Biomed Sci 13:813-823

Liu AH, Guo H, Ye M, Lin YH, Sun JH, Xu M, Guo DA (2007) Detection, characterization and identification of phenolic acids in Danshen using highperformance liquid chromatography with diode array detection and electrospray ionization mass spectrometry. J Chromatogr 1161:170-182

Liu J, Ha D, Xie Z, Wang C, Wang H, Zhang W, Zhang J, Chen S (2008) Ectopic expression of soybean GmKNT1 in Arabidopsis results in altered leaf morphology and flower identity. J Genet Genomics 35:441-449

Maghuly F, Leopold S, da Câmara MA, Borroto Fernandez E, Khan MA, Gambino G, Gribaudo I, Schartl A, Laimer M (2006) Molecular characterization of grapevine plants transformed with GFLV resistance genes: II. Plant Cell Rep 25:546-553

Michael AA, Anthony JP (2011) Activation tagging and insertional mutagenesis in barley. Methods Mol Biol 678:107-128

Nims E, Dubois C, Roberts SC, Walker EL (2006) Expression profiling of genes involved in paclitaxel biosynthesis for targeted metabolic engineering. Metab Eng 8:385-394

Sambrook J, Frisch EF, Maniatis T (1989) Molecular cloning: A laboratory manual (2nd ed). Cold Spring Harbor Press, New York, p 2344. ISBN 0-87969-136-0

Shu ZY, Zhang K, Ye XY, et al. (2010) Combination of naloxone, tanshinone and touch treatment on hypoxic ischemic encephalopathy of neonate. Res Int Trad Chin West Med 3:133-134

Takahashi K, Ouyang X, Komatsu K, Nakamura N, Hattori M, Baba A, Azuma J (2002) Sodium tanshinone IIA sulfonate derived from Danshen (Salvia miltiorrhiza) attenuates hypertrophy induced by angiotensin II in cultured neonatal rat cardiac cells. Biochem Pharmacol 64:745-749

Tani H, Chen X, Nurmberg P, Grant JJ, Maria MS, Chini A, Gilroy E, Birch PR, Loake GJ (2004) Activation tagging in plants: a tool for gene discovery. Funct Integr Genomics 4:258-266

Tsay HS, Ho HM, Gupta SK, Wang CS, Chen PT, Chen ECF (2012) Development of pollen mediated activation tagging system for Phalaenopsis and Doritaenopsis. Electron J Biotechnol. doi:10.2225/vol15-issue4-fulltext-1

Wang AM, Sha SH, Lesniak W, Schacht J (2003) Tanshinone (Salvia miltiorrhiza Extract) Preparations attenuate aminoglycoside-induced free radical formation in vitro and ototoxicity in vivo. Antimicrob Agents Chemother 47:1836-1841

Wang X, Wang Y, Jiang M, Zhu Y, Hu L, Fan G, Li X, Gao X (2011a) Differential cardioprotective effects of salvianolic acid and tanshinone on acute myocardial infarction are mediated by unique signaling pathways. J Ethnopharmacol 135:662-671

Weigel D, Ahn JH, Blazquez MA, Borevitz JO, Christensen SK, Fankhauser C, Ferrandiz C, Kardailsky I, Malancharuvil EJ, Neff MM, Nguyen JT, Sato S, Wang ZY, Xia Y, Dixon RA, Harrison MJ, Lamb CJ, Yanofsky MF, Chory J (2000) Activation tagging in Arabidopsis. Plant Physiol 122:1003-1013

Wu TW, Zeng LH, Fung KP, Wu J, Pang H, Grey AA, Weisel RD, Wang JY (1993) Effect of sodium tanshinone IIA sulfonate in the rabbitmyocardium and on human cardiomyocytes and vascular endothelial cells. Biochem Pharmacol 46:2327-2332

Wu CT, Mulabagal V, Nalawade SM, Chen CL, Yang TF, Tsay HS (2003) Isolation and quantitative analysis of cryptotanshinone, an active quinoid diterpene formed in callus of Salvia miltiorrhiza Bunge. Biol Pharm Bull 26:845-848 
Yagi A, Okamura N, Tanonaka K, Takeo S (1994) Effects of tanshinone VI derivatives on post-hypoxic contractile dysfunction of perfused rat hearts. Planta Med 60:405-409

Yan YP, Wang ZZ (2007) Genetic transformation of the medicinal plant Salvia miltiorrhiza by Agrobacterium tumefaciens-mediated method. Plant Cell Tiss Org 88:175-184

Yan Q, Hu ZD, Tan RX, Wu JY (2005) Efficient production and recovery of diterpenoid tanshinonein Salvia miltiorrhiza hairy root cultures with in situ adsorption, elicitation and semi-continuous operation. J Biotechnol 119:416-424

Yang R, Liu A, Ma X, Li L, Su D, Liu J (2008) Sodium tanshinone IIA sulfonate protects cardiomyocytes against oxidative stress-mediated apoptosis through inhibiting JNK activation. J Cardiovasc Pharmacol 51:396-401

Yoon Y, Kim YO, Jeon WK, Park HJ, Sung HJ (1999) Tanshinone IIA isolated from Salvia miltiorrhiza BUNGE induced apoptosis in HL60 human premyelocytic leukemia cell line. J Ethnopharmacol 68:121-127

Zhang Y, Song J, Zhao B, Liu H (1995) Crown gall culture and production of tanshinone in Salvia miltiorrhiza. Chin J Biotechnol 11:137-141

Zhang Y, Song J, Qi J, Lu G (1997) The plant regeneration of Salvia miltiorrhiza Bge. transformed by Agrobacterium. Zhongguo Zhong Yao Za Zhi (in Chinese) 22:274-275

Zhang C, Yan Q, Cheuk WK, Wu J (2004) Enhancement of tashinone production in Salvia miltiorrhiza hairy root culture by $\mathrm{Ag}+$ elicitation and nutrient feeding. Planta Med 70:147-151

Zhou L, Zuo Z, Chow MS (2005) Danshen: an overview of its chemistry, pharmacology, pharmacokinetics, and clinical use. J Clin Pharmacol 45:1345-1359

Zhou X, Chan K, Yeung JH (2012a) Herb-drug interactions with Danshen (Salvia miltiorrhiza): a review on the role of cytochrome P450 enzymes. Drug Metabol Drug Interact 27:9-18

Zhou X, Chan SW, Tseng HL, Deng Y, Hoi PM, Choi PS, Or PM, Yang JM, Lam FF, Lee SM, Leung GP, Kong SK, Ho HP, Kwan YW, Yeung JH (2012b) Danshensu is the major marker for the antioxidant and vasorelaxation effects of Danshen (Salvia miltiorrhiza) water-extracts produced by different heat waterextractions. Phytomedicine 19:1263-1269

doi:10.1186/1999-3110-54-37

Cite this article as: Ho et al: Activation tagging in Salvia miltiorrhiza can cause increased leaf size and accumulation of tanshinone I and IIA in its roots. Botanical Studies 2013 54:37.

\section{Submit your manuscript to a SpringerOpen ${ }^{\odot}$ journal and benefit from:}

- Convenient online submission

- Rigorous peer review

- Immediate publication on acceptance

- Open access: articles freely available online

- High visibility within the field

- Retaining the copyright to your article

Submit your next manuscript at $\gg$ springeropen.com 